\title{
Twinning in camels
}

\author{
Tinson A. H., K. S. Kuhad, K. Singh, R. Sambyal, A. Mugheiry, A. Rahman and J. \\ Al Masri \\ Scientific Center for Racing Camels, H.H. The Crown Prince Sheikh Khalifa bin \\ Zayed al Nahyan, P.O. Box 17292 Al-Ain, United Arab Emirates
}

\begin{abstract}
The camel has many unusual reproductive characteristics that contribute to making it a most unique animal. Pregnancy establishment occurs almost exclusively in the left horn and twin pregnancy is very rare. Even when embryos are transferred to the right horn migration occurs and the subsequent pregnancy is found on the left side. If multiple embryos are transferred together a twin pregnancy may establish but will only last for around 25 days as one pregnancy becomes dominant. This poster reviews reported cases of twins and looks at new techniques that will allow identical twin births to be more commonplace in the future.
\end{abstract}

Key words: pregnancy, twins, embryo transfer, camel.

\section{Introduction}

The camel has many unusual reproductive characteristics that contribute to making it a most unique animal. One of those unusual features is that pregnancy is almost exclusively carried in the left horn of the uterus and the live birth of twins is only rarely reported (Manefield and Tinson 1996). The anatomical differences between the sizes of the two horns and associated blood supply issues have been discussed as reasons for the low incidence. Regular ultrasonic examinations of camels suggest that the ovulation of two follicles simultaneously is not a rare event at the time of mating. Immunological factors could perhaps play a role if multiple ovulations led to non-identical twin pregnancies.

The separation of an embryo into two demi-embryos has been a common technique used to increase the embryo transfer pregnancy rate in cattle in recent times but the technique has so far proved impossible in the horse and very difficult in the camel. We have been trying to perfect the technique but despite different operators and techniques success, have been elusive.

\section{Natural Twins}

Incidence is extremely low and more often than not when twin pregnancies progress past 40 days abortion is more common than live birth. A report from
India described a particularly unusual set of twins of both sex born March 95 near Bikaneer. This event could not be remembered in the district for over $80 \mathrm{yrs}$ (Gohlt, 1995).

A pair of twin foetuses was reported aborted by a camel in Abu Dhabi by the local press earlier this year. In January 1999 we were called to attend a female camel who had aborted a set of quite advanced male twin calves and genetic testing revealed that they were indeed identical twin fetuses.

\section{Embryo Transfer}

With embryo transfer (E. T.) having become a more common technology in camels over the past 10 yrs one might have expected to have seen the birth of the odd set of twins. Superovulation and multiple embryos production would increase the opportunity to get the right conditions for twin pregnancies. Multiple embryo transfer to a single surrogate in our center has produced double pregnancies but while they can be confirmed by ultrasound at 18-25 days, they have generally reduced to a single dominant pregnancy by 40 days. Even when embryos are deliberately placed in the tip of the right horn during embryo transfer the pregnancy still establishes in the left after embryo migration has occurred and gives a similar result to embryos placed directly in the left horn (McKinnon and Tinson 1992). 


\section{Micromanipulation}

Morulas have consistently given poor pregnancy rates in embryo transfer in camels. In earlier work morulae and blastocyts were reported as having a lower pregnancy rate than hatched blastocyst which was surprising at the time given the delicate nature of the hatched structure compared to structures in zonas in other species (McKinnon, et al,1994). As overall pregnancy rates for E.T. in camels approached $60 \%$ it was noticed that morula transfer rates still stayed around $25 \%$ suggesting that when morulas were present in a flush it was possible that some of them had arrested in their development (Tinson 1996).

These observations took our focus on freezing and micro-manipulation techniques away from the smaller structures in zonas and placed our focus to the hatched blastocysts especially in regards to attempts to split embryos. Hatched blastocysts still have a clearly visible inner cell mass and are the easiest to handle of the hatched embryos. While it is possible to use protein free splitting solutions and electrostatic attraction to biopsy camel embryos (Harrison, 1998) this is not the case when trying to position the structure for an attempted twin split.

The use of new Eppendorf "robotic arms" for sperm injection techniques and micromanipulation opened new possibilities in the area of embryo separation. A special holding pippette was designed and trialed for holding embryos in place to allow either biopsy or splitting of camel embryos. Camels flushed 7 days post mating were searched for the presence of hatched blastocysts and 6 were collected. An AB Technology splitting blade was fitted to the sperm injection arm of the Eppendorf (Germany) equipment and the embryos were put in holding solution and placed with the new holding pipette so the inner cell mass was clearly visible. The embryos were sectioned in half and then the 12 demi-embryos were transferred to surrogates. Two weeks later the camels were scanned and the results appear in table 1 . unfortunately one of the twin pregnancies resorbed and another aborted later in the pregnancy so we had to repeat the process till we achieved the birth of the twins in March 2001.

\section{Future}

Like all facets of embryo transfer in the camel, the twinning results will improve with time. The advantages with increased pregnancy rates per embryo collected will be very important in valuable animals especially those that give low numbers of

Table1. Results of 6 twin split embryo transfers of 6 hatched blastocysts resulting in two sets of identical twin pregnancies and a pregnancy rate of $100 \%$ per embryo split

\begin{tabular}{ccccccc}
\hline Father & Mother & Embryo & Split & Recipient & Result & Comments \\
\hline & & G1.5HB & $942 \mathrm{~A}$ & 114 & Positive & Identical Twin \\
& & $942 \mathrm{~A}$ & 179 & Positive & Pregnancies \\
& & G1.5HB & $942 \mathrm{~B}$ & 106 & Positive & \\
SHAHEEN & $\mathbf{9 4 2}$ & G1.5HB & $942 \mathrm{C}$ & 189 & Negative & \\
& & & $942 \mathrm{C}$ & 170 & Positive & \\
& & \multirow{2}{*}{ G1.5HB } & $942 \mathrm{D}$ & 893 & Negative & \\
HAMLOOL & \multirow{296}{*}{ G1.5 HB } & $986 \mathrm{~A}$ & 379 & Positive & Identical Twin \\
MORGEER & & & $986 \mathrm{~A}$ & 232 & Positive & Pregnancies \\
& & \multirow{2}{*}{ G1.5 HB } & $986 \mathrm{~B}$ & 337 & Negative & \\
& & & $986 \mathrm{~B}$ & 248 & Negative & \\
\hline
\end{tabular}


embryo recovery. With the success of embryo biopsy and sexing it will be possible to sex and twin embryos in the near future. This will be particularly relevant when single cell biopsy techniques using direct aspiration systems become more developed.

The generation of more sets of racing camel twins will also allow us to look at many training and feeding aspects of the racing industry and further improve the performance results. Twining techniques could also have implications in breeding and preservation of threatened camel species such as the Wild Bactrian Camel of China and Mongolia.

\section{References}

Gahlot T. K. 1995. News Items. Journal Camel Practice and Research. p 159, Vol 2 No 2.
McKinnon A.O and A.H. Tinson, Embryo Transfer in Camels 1992. First International Camel Conference Dubai.. pp 203-208. Pub R\&W Publications UK.

Mc Kinnon A.O., A. H. Tinson and Nation 1994. Embryo Transfer in Dromedary Camels Theriogenology. 41 pp145-150.

Tinson A.H. 1996. Workshop in Camel Reproduction. June, Sydney 1996.

Harrison 1998. Personal communication.

Manefield G. and A. H. Tinson 1996. Camels a Compendium Vade Mecum Series C, No 22. P 87. Post Grad Foundation in Vet Science. Sydney Uni Press. 